\title{
A PLANE, ARCWISE CONNECTED AND CONNECTED IM KLEINEN POINT SET WHICH IS NOT STRONGLY CONNECTED IM KLEINEN*
}

\author{
BY R. L. WILDER
}

In a recent paper, $\nmid \mathrm{G}$. $\mathrm{T}$. Whyburn gave an example, in threedimensional space, of an arcwise connected and connected im kleinen point set which is not arcwise connected im kleinen, and raised the question as to whether such a set can exist in the plane; also, whether there exists in the plane a strongly connected and connected im kleinen set which is not strongly connected im kleinen. The purpose of this note is to answer both of these questions affirmatively. Indeed, there exists in the plane a point set $M$ which is arcwise connected and connected im kleinen, but which fails at every point to be strongly connected im kleinen.

Let $S$ denote the set of all points of the plane that lie within or on the square whose vertices are the points $(0,0),(1,0),(1,1)$, $(0,1)$; denote these vertices by $A, B, C, D$, respectively. The open interval $\langle A B\rangle$ is the sum of $c$ (where $c$ is the cardinal number of the continuum) mutually exclusive sets each of which is dense in $A B$. $\ddagger$ Denote the class of these sets by $X$.

Let $K$ denote the class of all continua contained in $S$, which contain at least one point of each of the straight line intervals $A D$ and $B C$, but no point of $A B$ or $C D$. This class has the cardinal number $c$.

There exists a one-to-one correspondence, $T$, between the elements of the classes $X$ and $K$. Let $E(X)$ and $E(K)$ be elements of $X$ and $K$, respectively, which correspond to one another under $T$. Then, if $x$ is a point of $E(X)$, denote by $l_{x}$ the set of points of $S$ that lie on the vertical line through $x$, and let $P_{x}$ denote the

\footnotetext{
* Presented to the Society, December 31, 1930.

$\dagger$ Concerning points of continuous curves defined by certain im kleinen properties, Mathematische Annalen, vol. 102 (1929), pp. 313-336.

† See Knaster and Kuratowski, Sur les ensembles connexes, Fundamenta Mathematicae, vol. 2 (1921), pp. 206-255; see especially p. 252. This paper will be referred to hereafter as S.E.C.
} 
first point (in ascending order of ordinates) of $E(K)$ on $l_{x}$. The set of all points $P_{x}$ obtained from $E(K)$ in this way denote by $N_{E(X)}$. Then let $N=\sum_{E(X)} N_{E(X)}$, and let $M=S-N$.

The set $N$ is punctiform.* Also, the set $M$ is obviously arcwise connected; for if $P$ and $Q$ are points of $M$, there is a broken line of $M$ connecting $P$ and $Q$, consisting of portions of the broken line $A B C D A$ and of the vertical lines through $P$ and $Q$. Furthermore, $M$ is connected im kleinen. Suppose, for instance, $P$ is a point of $M$ lying within the square $A B C D$. Let $S(P)$ denote a circle with center at $P$ and lying wholly within $S$, and let $R$ denote the set of all points of $M$ that lie within $S(P)$. Then $R$ is connected. For suppose $R$ is the sum of two mutually separated sets $R_{1}$ and $R_{2}$. Then there exists $\dagger$ a continuum $F$ which separates the plane between two points $P_{1}$ and $P_{2}$ belonging to $R_{1}$ and $R_{2}$, respectively, and which contains no point of $R$. As $F$ has points within $S(P)$, there is a subcontinuum $F^{\prime}$ of $F$ which lies wholly within $S(P)$ and is therefore a subset of $N$. As $N$ is punctiform, this is of course impossible. Consequently $R$ is connected, and $M$ is connected im kleinen.

It remains only to observe that $M$ is not strongly connected im kleinen at any point. Suppose, for instance, $P$ is a point of $M$ within $A B C D$. Define $S(P)$ as above, and let $S^{\prime}(P)$ be a circle concentric with and lying within $S(P)$. Let $Q$ be a point of $M$ interior to $S^{\prime}(P)$, but not on the same vertical as $P$. A continuum $H$ joining $P$ and $Q$ and interior to $S(P)$ belongs to some $E(K)$ of such a character that $H$ contains points of $N$. Consequently $M$ is not strongly connected im kleinen at $P$. If $P$ lies on $A B C D A$, say on $A B$, we choose $Q$ so that the point of $N$ on the vertical through $Q$ lies below $Q$, and employ a suitably chosen subcontinuum $H^{\prime}$ of $H$ that does not meet $A B C D A$.

The University of Michigan

* See S.E.C., pp. 245-246.

$\dagger$ See S.E.C., Theorem 37. 